\title{
High-intensity interval training: a review of its impact on glucose control and cardiometabolic health
}

\author{
Sophie Cassidy ${ }^{1} \cdot$ Christian Thoma $^{2} \cdot$ David Houghton $^{1} \cdot$ Michael I. Trenell ${ }^{1}$
}

Received: 26 April 2016 / Accepted: 17 August 2016 / Published online: 28 September 2016

(C) The Author(s) 2016. This article is published with open access at Springerlink.com

\begin{abstract}
Exercise plays a central role in the management and treatment of common metabolic diseases, but modern society presents many barriers to exercise. Over the past decade there has been considerable interest surrounding high-intensity interval training (HIIT), with advocates claiming it can induce health benefits of similar, if not superior magnitude to moderate-intensity continuous exercise, despite reduced time commitment. As the safety of HIIT becomes clearer, focus has shifted away from using HIIT in healthy individuals towards using this form of training in clinical populations. The continued growth of metabolic disease and reduced physical activity presents a global health challenge and effective therapies are urgently required. The aim of this review is to explore whether the acclaim surrounding HIIT is justified by examining the effect of HIIT on glucose control, its ability to affect cardiovascular function and the underlying mechanisms of the changes observed in those with common metabolic diseases. It also explores translation of the research into clinical practice.
\end{abstract}

Keywords Cardiovascular system - Exercise $\cdot$ Exercise therapy $\cdot$ Metabolic diseases $\cdot$ Metabolism $\cdot$ Physical fitness . Review $\cdot$ Weight loss

Michael I. Trenell

michael.trenell@ncl.ac.uk

1 MoveLab, Institute of Cellular Medicine, The Medical School, Newcastle University, 4th Floor William Leech Building, Framlington Place, Newcastle upon Tyne NE2 4HH, UK

2 School of Interprofessional Health Studies, Auckland University of Technology, Auckland, New Zealand

$\begin{array}{ll}\text { Abbreviations } \\ \text { EDV } & \text { End-diastolic volume } \\ \text { FMD } & \text { Flow mediated dilation } \\ \text { HIIT } & \text { High-intensity interval training } \\ \mathrm{HR}_{\max } & \text { Maximum heart rate } \\ \text { MICT } & \text { Moderate-intensity continuous training } \\ \text { NAFLD } & \text { Non-alcoholic fatty liver disease } \\ \text { PGC-1 } \alpha & \text { Peroxisome proliferator-activated receptor } \\ & \text { gamma, coactivator 1, alpha } \\ \text { RPE } & \text { Rate of perceived exertion } \\ \dot{V} \mathrm{O}_{2 \text { max }} & \text { Maximal oxygen consumption } \\ \dot{V} \mathrm{O}_{2 \text { peak }} & \text { Peak oxygen consumption }\end{array}$

\section{Why exercise?}

Before the agricultural, industrial and digital ages, humans expended large amounts of energy in activities centred on maintaining shelter and procuring food and water [1]. Fast forward some 350 generations and the barriers to exercise and physical activity in the 21 st century are enormous. Sedentary behaviours, such as the use of mechanised transport and screen-based leisure pursuits have become the norm in modern society. There is an urgent need therefore to find practical, attractive and effective exercise therapies to combat the wave of inactivity sweeping through the western world.

Not only is exercise part of our nature, it is strongly associated with reduced chronic disease risk. Globally, metabolic disorders such as the metabolic syndrome, non-alcoholic fatty liver disease (NAFLD), type 2 diabetes and the closely associated cluster of cardiovascular diseases are rapidly increasing [2]. European and US treatment algorithms for these obesity driven epidemics recommend weight loss and maintenance as a main priority across all stages $[3,4]$. Conceivably, this can be achieved through energy restriction and/or physical exercise. 
Current management guidelines for these common metabolic conditions advise individuals to undertake around $150 \mathrm{~min}$ of moderate-to-vigorous aerobic exercise per week, spread over most days of the week, in addition to resistance training on at least 2 days of the week $[5,6]$. The emphasis remains on moderate-intensity continuous training (MICT); however there is mounting evidence that high-intensity interval training (HIIT) provides an alternative means of achieving the same or greater health benefits vs MICT, provided there are no medical contraindications to engaging in HIIT and that it is well tolerated and preferred by the individual taking part. We refer readers to recent meta-analyses for a comprehensive analysis of the metabolic [7] and cardiorespiratory [8] benefits of HIIT in patient groups. The aim of this review is to assimilate existing evidence and provide a clinically relevant narrative of the cardiometabolic benefits of HIIT in those with common metabolic diseases, before moving onto discussing its safety profile, tolerability and practical considerations for translation into clinical care. The information presented in this review is not part of a formal systematic review and, therefore, may not have been subjected to the rigor required for such a summary of the data currently available on HIIT.

\section{What is HIIT?}

HIIT can be described as 'brief intervals of vigorous activity interspersed with periods of low activity or rest', which induces a strong acute physiological response (Fig. 1) [9]. A number of HIIT protocols have been adopted in the literature (see Table 1), but the majority of interventions use highintensity intervals lasting between 1 and $4 \mathrm{~min}$. The goal of HIIT is to accumulate activity at an intensity that the participant would be unable to sustain for prolonged periods (i.e. 80 $95 \%$ of peak oxygen consumption $\left(\dot{V} \mathrm{O}_{2 \text { peak }}\right)$ or $>90 \%$ of maximum heart rate $\left(\mathrm{HR}_{\max }\right)$, therefore the recovery time should be sufficient to allow the subsequent interval to be completed at the desired intensity. The total duration of a HIIT session tends to be $\geq 20 \mathrm{~min}$, which actually makes it comparable with recommendations for MICT, in terms of duration. There is also a sub-category of HIIT involving 10-30 second intervals and intensities often exceeding $100 \%$ $\dot{V} \mathrm{O}_{2 \text { peak }}$, i.e. 'all-out' exercise at a workload that is above maximal aerobic capacity [10]. This is often called sprint interval training and has not been substantially tested in clinical populations and will therefore not be covered further.

The vast majority of the published HIIT research, particularly in clinical populations, has used exercise modalities involving cycling, walking, and running, mostly carried out on stationary cycles and treadmills (see Table 1). However, other equipment, such as cross-trainers/ellipiticals [11], are reasonable options for some. Evidently there is clear variation throughout the literature and it still remains to be determined whether an optimal HIIT protocol exists for metabolic disease management.

\section{HIIT and metabolic health}

\section{Skeletal muscle molecular adaptations}

A number of molecular adaptations have been identified within skeletal muscle following HIIT (Fig. 2). Skeletal muscle is the primary site for glucose disposal via insulin- and noninsulin-mediated glucose uptake; the latter stimulated by muscular contraction. It therefore plays a large role in regulating metabolism.

Increased GLUT-4 content GLUT-4 content in the vastus lateralis increased by $369 \%$ following six sessions of HIIT in type 2 diabetes patients [12]. Insulin resistance underlies metabolic disease and although decreased GLUT- 4 content is
Fig. 1 An example of a HIIT protocol. Schematic of the HIIT protocol adopted by our group in adults with NAFLD [39] and type 2 diabetes [38]. Intensity was based upon the perceived rate of exertion (RPE), inducing a strong acute physiological response in heart rate (shown as \% peak heart rate $\left.\left[\mathrm{HR}_{\text {peak }}\right]\right)$, which increases across intervals

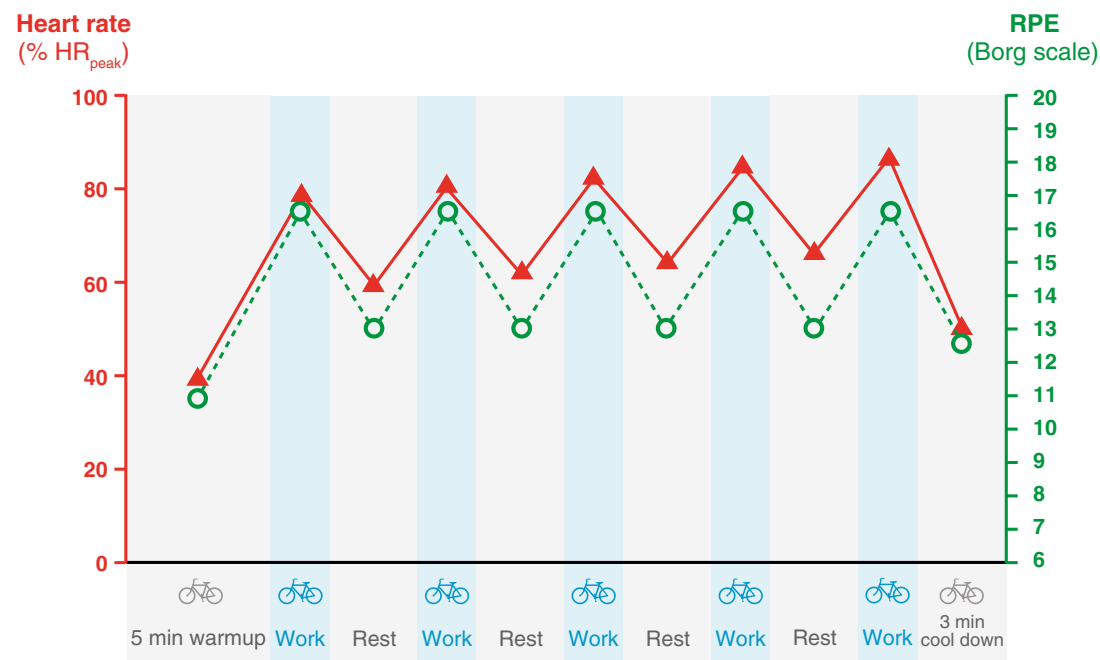




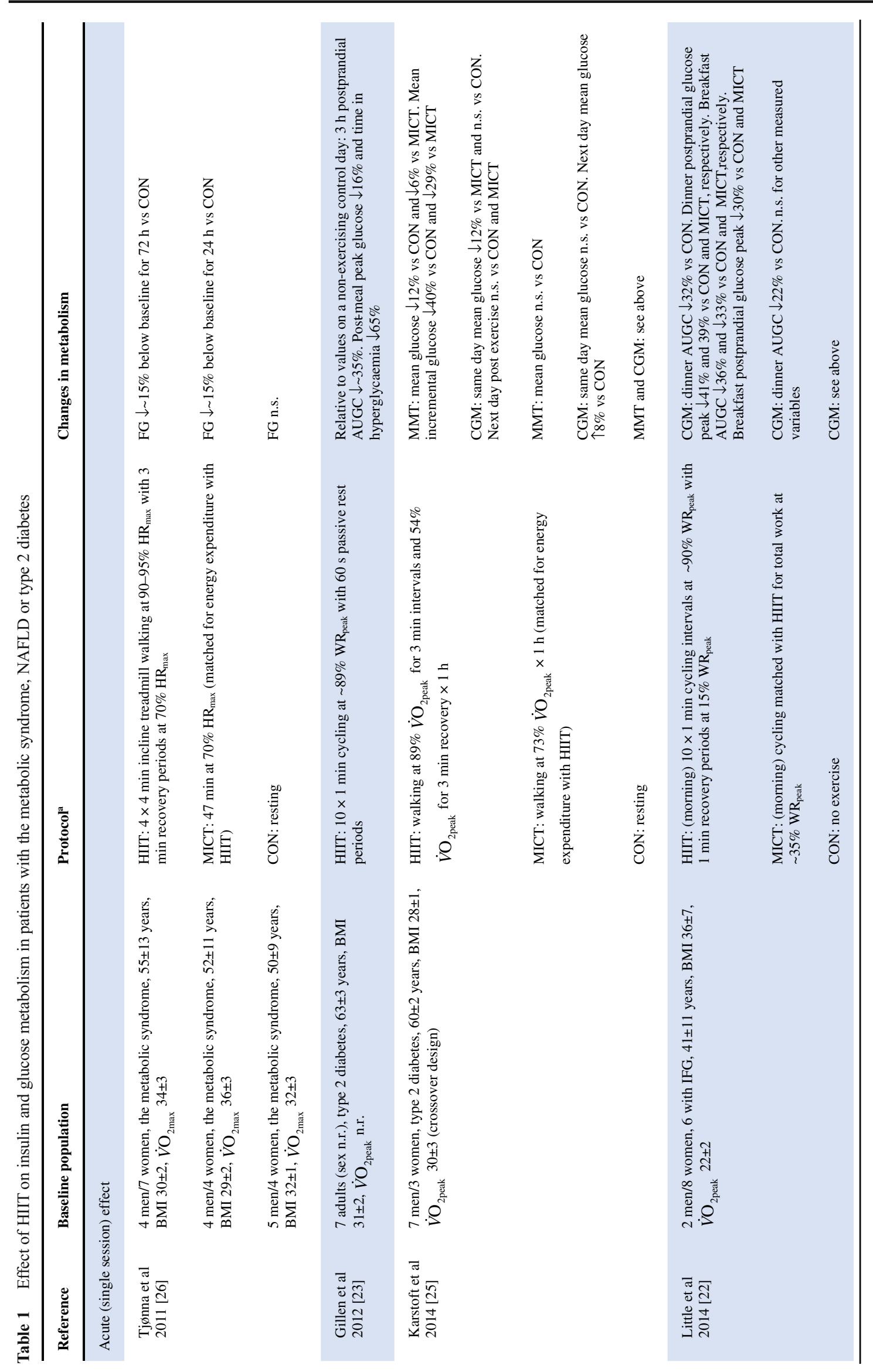




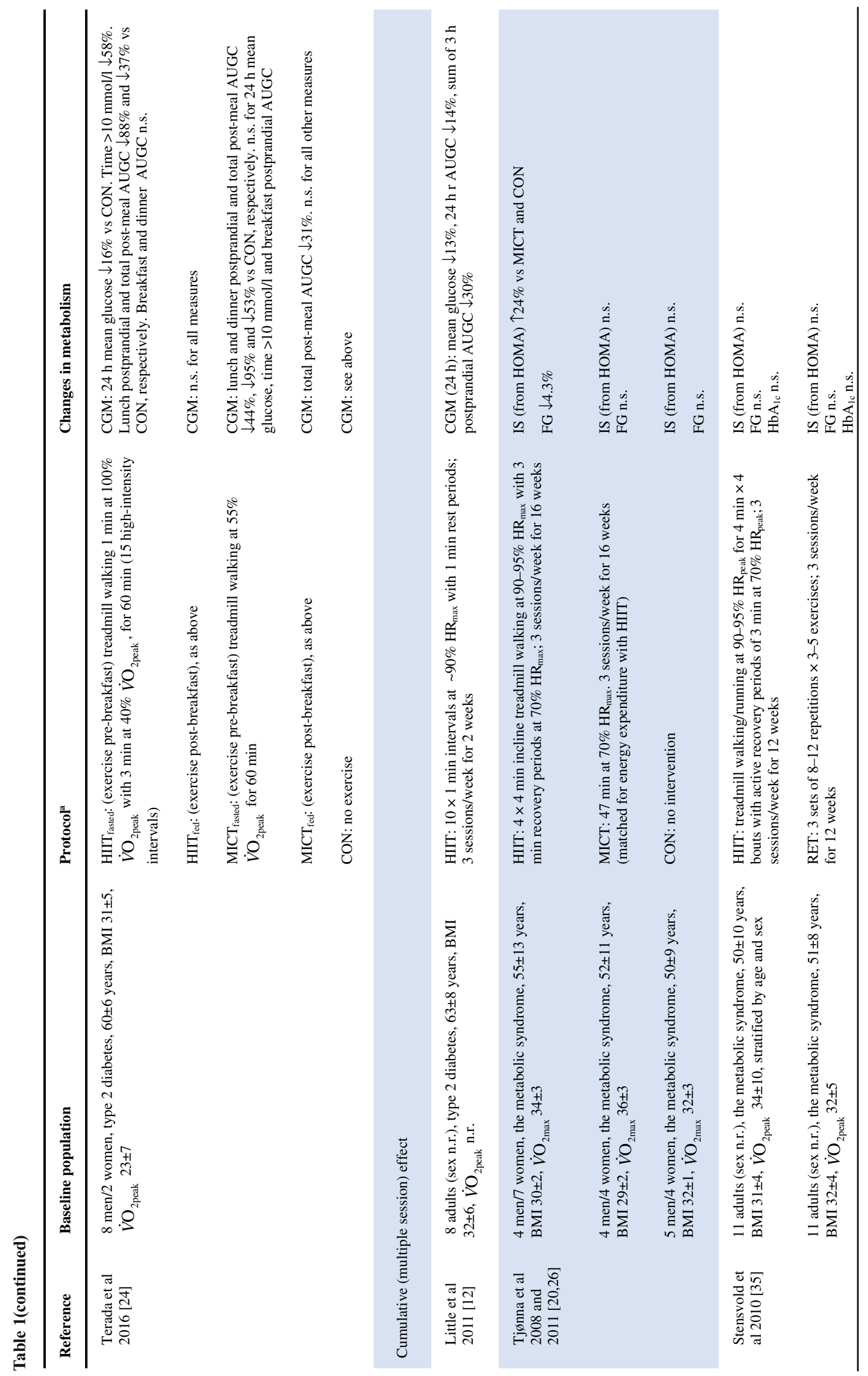




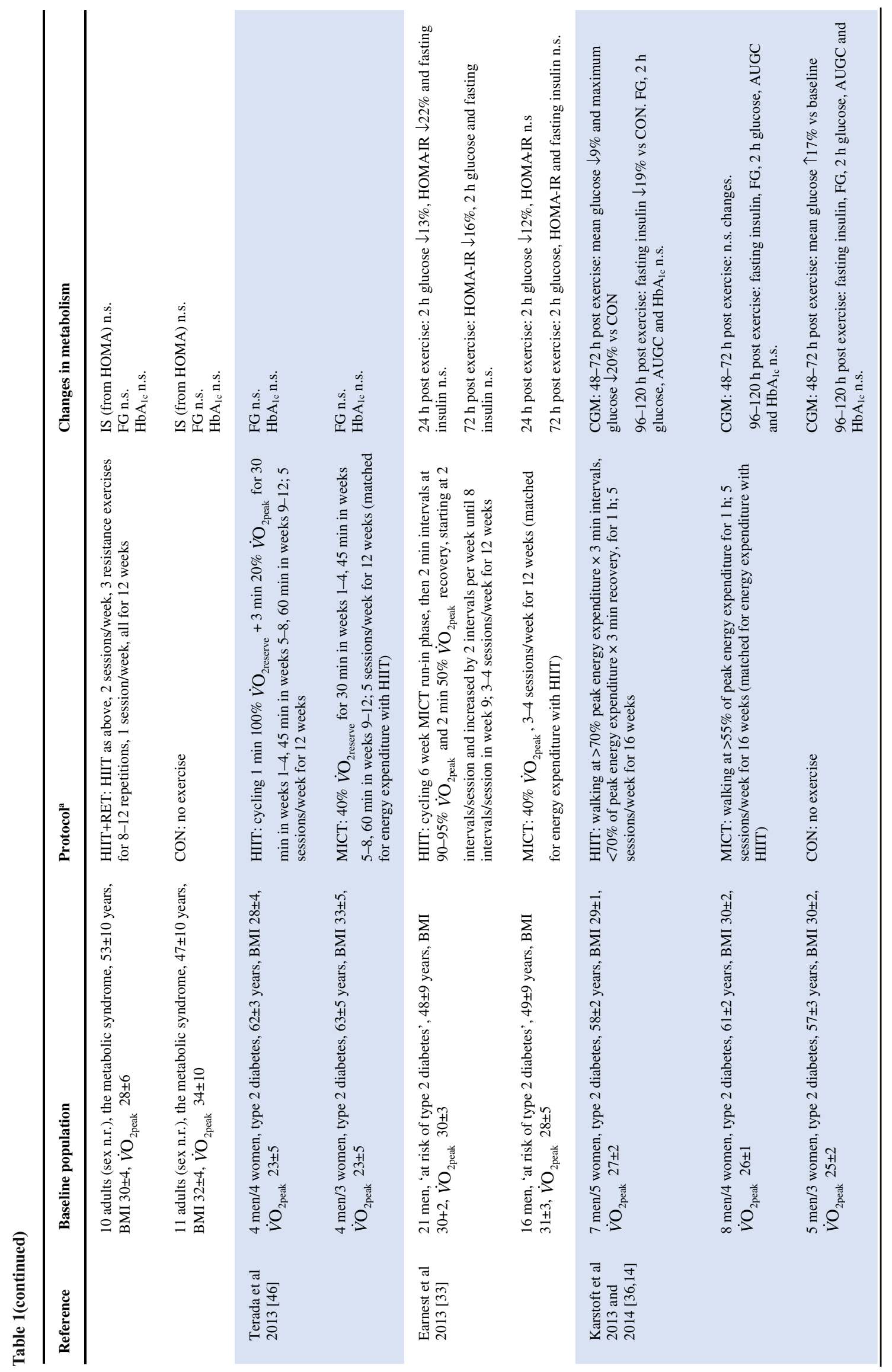




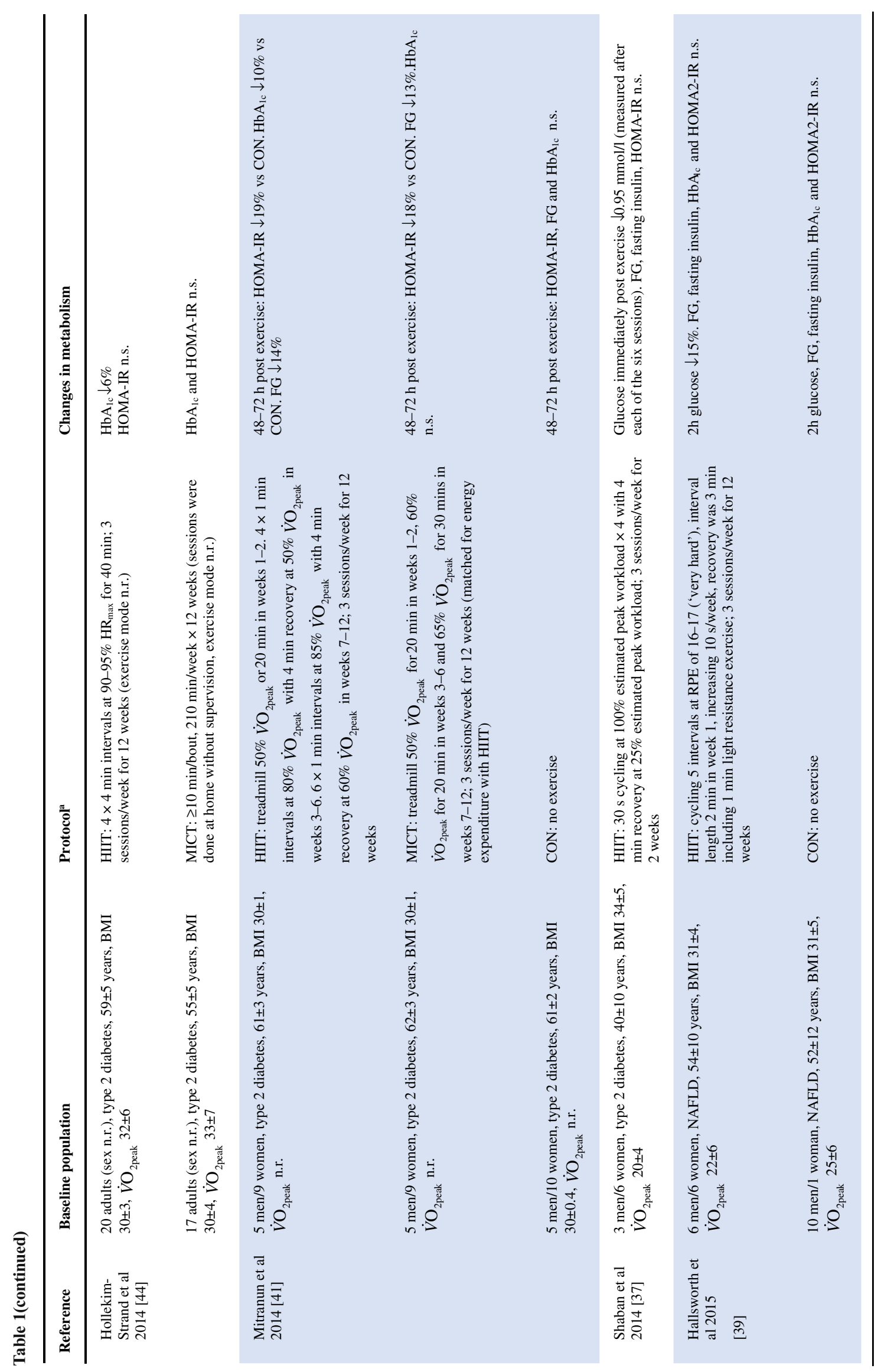




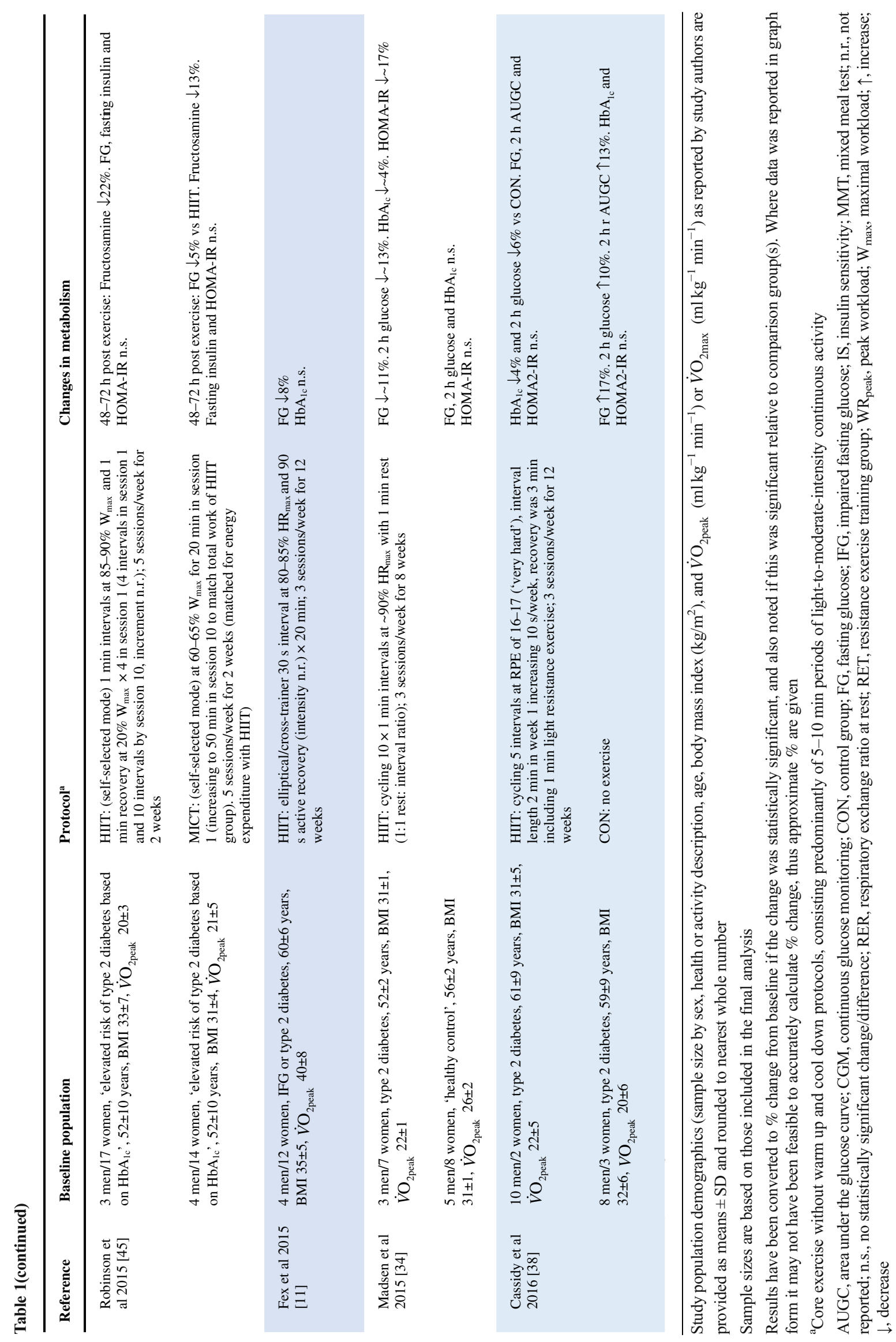


Fig. 2 Cardiometabolic effects of HIIT. The figure depicts the previously reported muscular and cardiovascular impact of HIIT in those with common metabolic diseases. In boxes of text: upward arrow, increase; downward arrow, decrease. EDV, end diastolic volume; $\mathrm{EF}$, ejection fraction; FMD, flow mediated dilation; SR, sarcoplasmic reticulum; SV, stroke volume

\section{Cardiometabolic effects of HIIT}

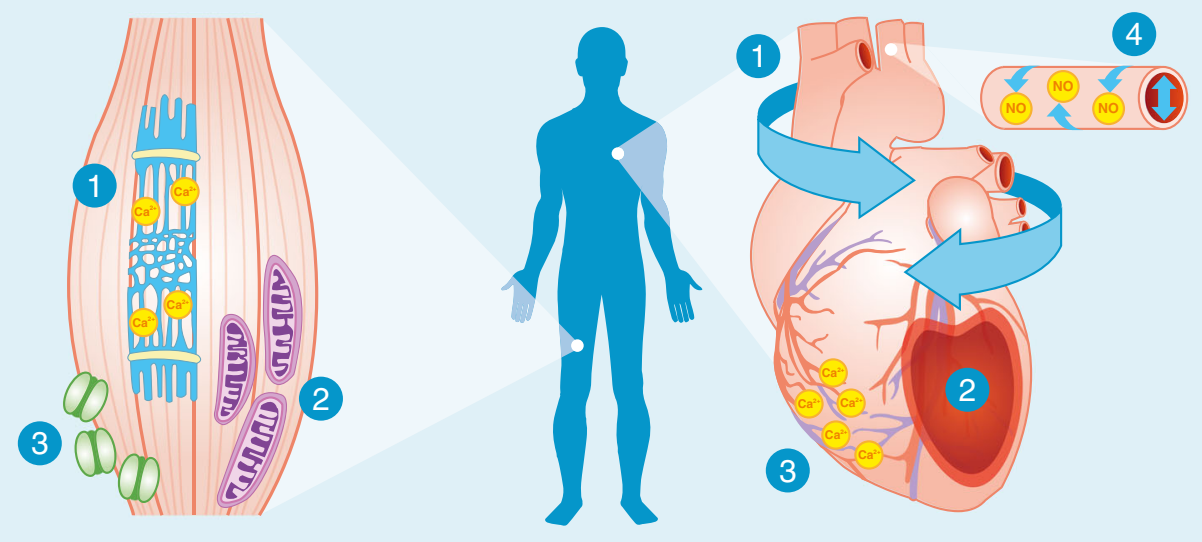

\section{Changes in skeletal muscle}

(1) $\mathrm{Ca}^{2+}$ reuptake into $\mathrm{SR}$ 會 $=$ muscle work capacity 1

(2) Mitochondria biogenesis = oxidative capacity

3 GLUT4 會 $=$ glucose transport 1

\section{Changes to cardiovascular system}

(1) Torsion $v=$ myocardial damage

(2) EDV 슬 $=$ preload 술

(3) $\mathrm{Ca}^{2+}$ handling $\mathrm{A}=\mathrm{SV}$ 슬 $+\mathrm{EF}$ 술

(4) $\mathrm{FMD}$ 合 $=\mathrm{O}^{2}$ supply 命 not the cause of insulin resistance, any increase in this protein improves glucose transport within skeletal muscle [13]. Another study found that 16 weeks of HIIT in type 2 diabetes patients induced higher membrane-bound GLUT-4 and GLUT-4 mRNA levels in comparison with energy matched MICT, but no rise in overall GLUT-4 protein content was observed [14]. The reduced intensity of intervals in this study ( $70 \% \dot{V} \mathrm{O}_{2 \text { peak }}$ ) compared with other HIIT protocols is worth noting, however, as is the fact that biopsies were obtained 5-6 days post-training.

Mitochondrial adaptations Reduced mitochondrial content [15], mitochondrial function [16] and markers of mitochondrial biogenesis in skeletal muscle are commonly observed in individuals with metabolic disease [17] and have been suggested to contribute to insulin resistance. In adults with type 2 diabetes, 2 weeks of HIIT $\left(90 \% \mathrm{HR}_{\max }\right)$ significantly increased mitochondrial capacity evidenced by an increase in citrate synthase activity and raised content of electron transport chain complexes [12]. In contrast, 16 weeks of interval walking $\left(70 \% \dot{V} \mathrm{O}_{2 \text { peak }}\right)$ only elicited changes in citrate synthase mRNA expression but not in citrate synthase activity. However, as stated above, this could be due to the lower intensity of intervals used in this study compared with traditionally adopted HIIT protocols and because muscle biopsies were obtained 5-6 days post-training [14].

Peroxisome proliferator-activated receptor, gamma, coactivator 1 , alpha (PGC-1 $\alpha$ ) regulates muscle mitochondrial biogenesis [18]. Following HIIT, increases in nuclear PGC-1 $\alpha$ levels have been observed [19], as well as increases in total PGC- $1 \alpha$ vastus lateralis content in biopsy samples. Similar changes were not observed following energy matched MICT [20]. It has been proposed that the fluctuations in ATP turnover during interval training, which differs from the usual steady state conditions of ATP production, could activate signalling pathways that lead to this increase in PGC- $1 \alpha$ following HIIT [21].

Sarcoplasmic reticulum Sarcoplasmic reticulum $\mathrm{Ca}^{2+}$ handling plays an important role in muscle fatigue and increased $\mathrm{Ca}^{2+}$ reuptake into the sarcoplasmic reticulum has been demonstrated following HIIT, but not MICT in adults with the metabolic syndrome [20]. Increases as large as 50-60\% were observed in $\mathrm{Ca}^{2+}$ reuptake, significantly improving the work capacity of the muscle and thereby contributing to improvements in fitness following HIIT. This study indicates that HIIT provides a more potent stimulus compared with MICT to induce skeletal muscle adaptations. However, definite conclusions cannot be drawn from one small study, with a total sample size of 32 participants.

\section{HIIT and glucose control}

Key studies on the impact of HIIT on glucose control have been summarised in Table 1. These adopt a range of protocols and cover both the acute and training responses to HIIT. 
The acute response Few studies have assessed the acute response to HIIT in patients with metabolic disorders and those that have are summarised in Table 1. Relative to no exercise, a single session of HIIT reduces same-day postprandial area under the glucose curve in those with impaired fasting glucose [22] or type 2 diabetes [23, 24]. Similarly, HIIT is associated with reduced time of glucose being $\geq 10 \mathrm{mmol} / \mathrm{L}$ $[23,24]$. However, studies assessing the effect of HIIT on mean $24 \mathrm{~h}$ glucose levels have been less consistent, with some indicating no effect $[22,23,25]$, and one showing a reduction but only when the exercise was performed in a fasted state [24]. When compared with energy matched MICT, HIIT tended to be slightly superior $[22,25,26]$ (see Table 1 ). As measurements were not reported much past 24 hours post exercise, the duration of these effects is uncertain. Earlier work relying on changes in fasting glucose to assess impact, suggests a measurable effect may last as long as $72 \mathrm{~h}$ post HIIT, but for a shorter period following MICT [26].

Since postprandial glucose excursions are strong predictors of cardiovascular disease [27], which may be due to possible inductions of oxidative stress and micro/macrovascular damage [28], the above findings are of clinical relevance. Unfortunately, the available research does not provide clarity on dose-response in terms of intensity, duration or total energy expenditure. Further head-to-head comparisons of different protocols are required. However, it is worth noting that a protocol of 1 min intervals with 1 min recovery, repeated ten times (a modest time investment), improves acute glucose control [22, 23].

The transient nature of changes in glucose metabolism in response to exercise is well documented. Insulin-independent glucose disposal is increased during and for approximately $60 \mathrm{~min}$ post exercise [29]. Insulin-dependent glucose disposal increases for several hours to a few days following exercise $[29,30]$. These effects are localised to contracting muscle [29], thus exercise involving a larger muscle mass is preferable. Higher-intensity exercise has been shown to recruit a larger proportion of muscle fibres compared with moderateintensity exercise [31], which may explain greater improvements in glucose regulation following HIIT. In light of these acute adaptations, patients should be recommend not to have more than two exercise-free days, in accordance with guidelines [32].

The training response Of the studies assessing the effects of longer-term HIIT ( $\geq 2$ weeks), some report reduced fasting glucose $[11,20,26,33,34]$, while others report no change [35-39] (Table 1). Where reductions in fasting glucose are observed, they appear to be similar to those seen following MICT [7]. Fasting glucose is predominantly a marker of hepatic insulin sensitivity. After just 1 week of a diet very low in energy (very low calorie diet; $2510 \mathrm{~kJ} /$ day [600 kcal/ day]), liver fat content decreased by $30 \%$, hepatic insulin sensitivity significantly improved and fasting glucose fell by $35 \%$ in adults with type 2 diabetes [40]. The reduction in fasting glucose following participation in HIIT is generally smaller [11, 20, 26, 34, 41], $(\leq 14 \%$, see Table 1$)$, suggesting that exercise (whether HIIT or MICT), lacks potency for improving hepatic insulin sensitivity, when compared with consumption of a very low calorie diet. This is most likely because exercise elicits a smaller energy deficit than that achieved with a modest change in eating behaviour. For example, to achieve an energy deficit similar to that achieved by reducing energy intake by the equivalent of the energy in a blueberry muffin ( 1891 kJ [452 kcal]), a $68 \mathrm{~kg}$ female would need to run approximately $38 \mathrm{~min}$ at a pace of $9.7 \mathrm{~km} / \mathrm{h}$ [42]. We did, however, show that HIIT was able to significantly reduce liver fat and, thereby, fasting glucose in some type 2 diabetes individuals [38], but the average reduction in liver fat did not result in a significant reduction in fasting glucose levels. Whether an increase in the duration of the HIIT intervention (i.e. $>12$ weeks) would achieve a reduction in fasting glucose levels in this cohort is yet to be determined.

HIIT has been shown to improve peripheral insulin sensitivity in those with impaired metabolic control. The molecular adaptations to HIIT described above, including raised GLUT-4 content, increased aerobic enzyme capacity and mitochondrial biogenesis, have all been associated with improved peripheral insulin sensitivity $[13,43]$. Studies assessing the metabolic impact of HIIT in those with common metabolic diseases have found no change in HOMA-IR [37-39, 44, 45], whereas others have shown an approximate $20 \%$ improvement compared with a control group [20, 33, 34, 41] (Table 1). When compared with MICT, HIIT seems to have a small but significant benefit on insulin resistance [7].

HIIT can also decrease $\mathrm{HbA}_{1 \mathrm{c}}[34,38,41,44]$, yet some studies have reported no change $[11,36,39,46]$ (Table 1). Although there have been a number of studies published since, a meta-analysis found that a $0.47 \%$ absolute reduction in $\mathrm{HbA}_{1 \mathrm{c}}$ is observed with HIIT in adults with common metabolic diseases, compared with controls [7]. This is slightly lower than the $0.6 \%$ absolute $\mathrm{HbA}_{1 \mathrm{c}}$ reduction observed following aerobic and resistance exercise in type 2 diabetes [47]. Both HIIT and other forms of exercise compare well with improvements achieved through metformin [48] and are likely to have clinical benefits, since a $1 \%$ absolute rise in $\mathrm{HbA}_{1 \mathrm{c}}$ leads to a $21 \%$ increased risk of diabetes related death, a $14 \%$ increased risk of myocardial infarction and a $37 \%$ increased risk of myocardial infarction [47].

Other indicators of glucose control, such as $2 \mathrm{~h}$ glucose following an oral glucose challenge and glucose AUC are similarly inconsistent across studies (see Table 1). Several explanations for the reported inconsistencies across studies include differences in study populations, exercise protocols and the degree of volunteer supervision during exercise. However, the most plausible explanation is the variation in 
time of post-intervention measures relative to the last bout of exercise. Studies reporting both the acute and cumulative effect of HIIT have consistently shown that changes in indicators of glucose control last between 24 and $72 \mathrm{~h}$ post exercise $[25,26,33,36]$ (Table 1). Only one study has demonstrated a longer-term adaptation, in which fasting insulin was reduced 96-120 h post exercise [36]. Greater inter-study consistency in the timing of post exercise assessments is warranted in the future; continuous glucose monitoring for at least $72 \mathrm{~h}$ post exercise and $\mathrm{HbA}_{1 \mathrm{c}}$ assessments may also allow us to gauge benefit better.

Collectively, the improvements in glucose control following HIIT are clinically relevant but do not surpass those seen following the traditionally used MICT with regards to fasting glucose, $\mathrm{HbA}_{1 \mathrm{c}}$ and fasting insulin [7]. HIIT does seem to lead to greater improvements in peripheral insulin sensitivity [7], but overall the use of HIIT for improving glycaemic outcomes should not be over-emphasised compared with other forms of exercise training.

\section{HIIT and cardiovascular health}

Cardiovascular complications are the leading cause of mortality in those with common metabolic diseases [49, 50]. The interval design of HIIT to include rest periods enables patients to accumulate time at higher exercise intensities, thereby challenging the cardiovascular system. Limited evidence indicates that HIIT provides a stronger stimulus than MICT for eliciting myocardial improvements. Alongside the beneficial impact of HIIT on vascular and cardiorespiratory fitness, this suggests that the cardiovascular benefit of HIIT outweighs the metabolic benefit.

\section{Cardiac adaptations: molecular mechanisms}

Because of the difficulty of obtaining human myocardial tissue, most evidence for the molecular adaptations to high-intensity exercise comes from experimental rodent models, the hearts of which bear similarities to human hearts and mimic the human cardiac response to exercise training $[51,52]$. The $d b / d b$ mouse model provides a good representation of the human heart in diabetic patients. Following 13 weeks of HIIT, contractility and $\mathrm{Ca}^{2+}$ handling were restored to normal levels as a result of raised transverse tubule (T-tubule) density, sarcoplasmic reticulum synchrony of $\mathrm{Ca}^{2+}$ release and sarcoplasmic reticulum $\mathrm{Ca}^{2+}$-ATPase (SERCA2a; $\mathrm{Ca}^{2+}$ transporter) activity [53]. These adaptations occurred despite no improvement in glucose or insulin levels, demonstrating the direct impact of HIIT upon the myocardium. Similar adaptations have been observed in heart failure and healthy rodent models [54, 55], with greater changes occurring following high-intensity exercise
(85-90\% maximal oxygen consumption $\left.\left[\dot{V}_{2 \max }\right]\right)$ compared with moderate-intensity exercise $\left(65-70 \% \dot{V}_{2 \max }\right)[55]$.

Exercise also activates the phosphoinositol-3 kinase/Akt/ mammalian target of rapamycin (mTOR) signal transduction pathway that leads to higher ribosomal biogenesis and protein synthesis, and thus induces physiological hypertrophy to a greater extent following high- $\left(85-90 \% \dot{V}_{2 \max }\right)$ vs moderate- $\left(65-70 \% \dot{V} \mathrm{O}_{2 \max }\right)$ intensity exercise $[52,55]$. The exercise-induced pathways activated in disease models may differ [54], but both healthy and disease rodent models indicate that exercise stimulates important transcriptional, translational and post-translational regulatory mechanisms that lead to structural remodelling of cardiac tissue and, thereby, improved strength of cardiac contractions [52].

\section{Cardiac structure}

Adults with common metabolic diseases display left ventricular concentric remodelling, which represents a reduction in end-diastolic volume (EDV) and is also known as pathological hypertrophy $[56,57]$. This reduction in EDV occurs in response to stress signals and is reflective of a build-up of collagen in the myocardium [58]. HIIT, on the other hand, has been shown to induce physiological hypertrophy [38], increasing left ventricular wall mass and EDV by means of a physiological response to growth signals [58]. The number of studies investigating cardiac structure following HIIT is small; our group showed an $8 \mathrm{ml}$ increase in EDV following 12 weeks of HIIT in type 2 diabetes patients [38], but no improvements in NAFLD patients [39]. Both of these studies compared HIIT with a non-exercise control, rather than MICT. That being said, HIIT has been shown to be superior to energy matched MICT in eliciting structural remodelling in those with hypertension [59] and heart failure [60].

\section{Cardiac function}

Systolic function Stroke volume and ejection fraction, two measures of the contractile capabilities of the heart, are reduced in those with metabolic disease [57]. Twelve weeks of HIIT induces systolic improvements in adults with type 2 diabetes [38, 44], hypertension [59] and heart failure [60]. Following 12 weeks of HIIT in heart failure patients, Wisløff et al [60] demonstrated a $35 \%$ and $17 \%$ relative increase in ejection fraction and stroke volume, respectively, but no change in these variables following energy matched MICT. These improvements are equal to those seen with commonly used prescription medications, such as ACE inhibitors or beta blockers [61]. Twelve weeks of HIIT in hypertensive patients improved early events in systole, which correlate to contractility and are load independent [59]. Furthermore, 12 weeks of 
HIIT in heart failure patients led to a $22 \%$ increase in global contractility [60]. These improvements were not observed following energy matched MICT [60].

Cardiac torsion describes the twisting motion of the heart during contraction and reflects the dominance of epicardial fibres over endocardial fibres. In adults with metabolic disease cardiac torsion is raised [62], reflecting damage to endocardial fibres. Interestingly, we observed reductions in cardiac torsion in adults with type 2 diabetes and NAFLD who partook in 12 weeks of HIIT, when compared with controls [38, 39], suggesting a reduction in endocardial damage following HIIT.

Diastolic function Diastolic dysfunction is often reported in those with common metabolic diseases [57, 63]. Impaired early filling of the left ventricle is indicative of stiffer, damaged myocardial fibres that are less compliant during relaxation; yet evidence suggests that HIIT has the capacity to target these abnormalities. Two studies have demonstrated significant improvements in early filling rates following 12 weeks of HIIT in adults with type 2 diabetes [38, 44], which were sustained 1 year later [44]. Similar diastolic improvements were also observed in adults with NAFLD [39]. These HIIT-induced elevations in early filling rate have been demonstrated to be as large as $49 \%$. In contrast, 12 weeks of MICT fails to have any impact upon diastolic variables [44, 59, 60]. These data suggest that exercise intensity is an important characteristic for inducing diastolic improvements. Diastolic dysfunction is an independent predictor of mortality [64], therefore any improvements in function are likely to be clinically significant.

\section{Vascular function}

Endothelial dysfunction is associated with metabolic disease [65] and considered one of the earliest pathophysiological processes in the progression to atherosclerosis. Flow mediated dilation (FMD) is a measure of endothelial dysfunction and is regulated by NO availability. In those with common metabolic disease, HIIT has been shown to be superior [44] or similar [35] to MICT for improving FMD. Although not limited to common metabolic diseases, a meta-analysis of 182 participants demonstrated twice the improvement in FMD following HIIT, compared with MICT [66]. This is most likely due to the greater shear stress experienced during higher-intensity exercise, since shear stress is the main stimuli for increasing NO availability in the endothelium [59]. Consequently, improved FMD results in greater perfusion and oxygen supply to peripheral tissue.

Findings with respect to the effect of HIIT on blood pressure in individuals with common metabolic diseases have been inconsistent; some studies demonstrate improvements $[11,20,35,45]$, whereas some show no change $[36,38,39,44]$ in blood pressure, despite positive cardiac remodelling [38]. Exercise guidelines for the treatment of hypertension advise low- to moderateintensity exercise [67], but these findings suggest further work is required to better define the role of HIIT in hypertension therapy.

\section{Skeletal muscle and cardiac adaptations combine to improve $\dot{V} \mathrm{O}_{2 \text { peak }}$ following HIIT}

It could be argued that the most important outcome following HIIT is cardiorespiratory fitness, as measured by $\dot{V} \mathrm{O}_{2 \text { peak }}$. Large prospective studies have demonstrated fitness to be more important than established risk factors for mortality [68], and low $\dot{V} \mathrm{O}_{2 \text { peak }}$ is independently associated with incident type 2 diabetes [69]. While the exerciseinduced increase in $\dot{V} \mathrm{O}_{2 \text { peak }}$ has never been directly linked to mortality, large scale studies indicate that improvements in fitness over time leads to significant reductions in mortality risk $[70,71]$.

$\dot{V} \mathrm{O}_{2 \text { peak }}$ is the gold standard measure of fitness and a strong indicator of how well the cardiac, pulmonary, vascular and peripheral systems are working together. A number of meta-analyses have demonstrated the substantial benefits of HIIT for $\dot{V} \mathrm{O}_{2 \text { peak }}$ and its superiority in comparison to MICT in healthy [72], coronary artery disease [73] and cardiometabolic disease patients [8]. In those with elevated cardiometabolic risk, the increase in $\dot{V} \mathrm{O}_{2 \text { peak }}$ with HIIT (19.4\%) was almost twice that of MICT (10.3\%) [8]. On average, $\dot{V} \mathrm{O}_{2 \text { peak }}$ increases by $5.4 \mathrm{ml} \mathrm{kg}^{-1} \mathrm{~min}^{-1}$ following HIIT, and even a smaller improvement of $3.5 \mathrm{ml} \mathrm{kg}^{-1} \mathrm{~min}^{-1}$ has been predicted to improve survival by $10-25 \%$ [74].

Figure 2 provides an overview of the skeletal muscle and cardiac adaptations that are likely to contribute to the improvements in $\dot{V} \mathrm{O}_{2 \text { peak }}$ observed with HIIT. As demonstrated in the figure, HIIT improves the capacity of both aspects of the oxygen supply and demand chain, but it is the cardiovascular adaptations in response to HIIT that are more likely to contribute to these $\dot{V} \mathrm{O}_{2 \text { peak }}$ improvements [75].

\section{HIIT and weight loss}

HIIT induces moderate weight loss $(0.5-4 \mathrm{~kg}$ reduction) in adults with common metabolic diseases [11, 20, 34, 36, 41, 46]. When compared with MICT, however, HIIT 


\section{Summary of HIIT}

Leads to modest improvements in metabolic control, of similar magnitude to other forms of exercise training

2 Should not be overstated for its role in weight loss

3 Has strong cardiovascular benefits

(4) Leads to large improvements in cardiorespiratory fitness, often superior to other forms of exercise training

provides no additional benefit as an exercise therapy for weight loss [7]. The ability of HIIT to induce reductions in body weight should therefore not be overstated in those with common metabolic diseases.

Although weight loss is strongly associated with reduced metabolic complications [76], it does not reflect changes in body composition; HIIT generally reduces whole body fat mass by $1-3 \mathrm{~kg}$, even when body weight remains stable [14, 35, 36, 41, 46, 77, 78]. Significant reductions in visceral and hepatic fat have also been shown with HIIT [14, 38, 39]. These findings are important since these fat depots increase cardiovascular disease risk [79], and metabolic dysfunction [40, 80]. Three possible mechanisms for HIIT-induced fat loss have been suggested:

1. increased mitochondrial density and capacity following HIIT leading to increased fat oxidation [81]

2. large elevations in catecholamines, which have been shown to drive lipolysis [82], especially in the abdominal tissue where there are significantly more $\beta$ adrenergic receptors, compared with subcutaneous fat [83]

3. appetite suppression: energy intake the day after HIIT was $\sim 1255 \mathrm{~kJ}$ (300 kcal) lower than after MICT, and $\sim 2510 \mathrm{~kJ}$ (600 kcal) lower than after rest [84]

It remains unclear whether HIIT is superior to MICT for fat loss, with some studies supporting this notion [14, $36,46]$ and some not $[34,35,41,45,78]$. To date, evidence to support HIIT over other types of exercise for the management of body fat levels is unfounded, but there is enough proof to suggest that HIIT can induce positive changes in body composition in adults with common metabolic diseases.

A summary of the effects of HIIT can be found in the text box 'Summary of HIIT'.

\section{Is HIIT safe?}

Given the strong cardiovascular-focused physiological response to HIIT, it is appropriate to define the safety of high-intensity activity in those at elevated cardiometabolic risk. The acute cardiac response to HIIT has been assessed in a few studies. In patients with coronary heart disease, no contraindications to HIIT were observed and undesirable changes, such as ST-segment depression, recovered during interval recovery periods $[85,86]$. Also, in patients with chronic heart failure, cardiac stress (as assessed by rate pressure product) stayed within acceptable values [87]. Furthermore, the studies mentioned above did not report any serious adverse events with HIIT.

The largest available dataset assessing the safety of HIIT was derived from a clinical audit of 4846 cardiac rehabilitation patients. It identified two non-fatal cardiac arrests in 46,364 $\mathrm{h}$ of supervised HIIT, and one fatal cardiac arrest in $129,456 \mathrm{~h}$ of supervised MICT [88]. Although the low frequency of events makes the comparison between the two exercise modalities inconclusive, it also highlights that the risk of either approach is low. It is important to note that all patients were referred to cardiac rehabilitation by their general practitioner or hospital cardiologist and underwent a full medical screening and cardiopulmonary exercise test prior to taking part, to rule out recurrent ischaemia or chest pain during exercise.

The risk of sudden cardiac death and acute myocardial infarction is increased following vigorous activity in susceptible individuals, including those with structural heart disease and congenital complications [89]. The American College of Sports Medicine and the American Heart Association provide guidelines for identifying high risk patients and carrying out pre-exercise screening in such individuals $[6,89]$. According to these guidelines those with common metabolic diseases are automatically considered 'high risk'. On the whole, however, 


\section{Recommendations for HIIT prescription}

Frequency: 3 HIIT sessions per week

Intensity: Most easily measured by rating of perceived exertion (although may be variable in practice)

Time: Intervals should last between 1 and 4 minutes, with intervals at the shorter end being preferred by patients. The total time spent doing intervals should be 10-20 minutes per session

Type: Activities involving a large muscle mass

mounting clinical evidence supports HIIT as a safe therapy for the majority of individuals with elevated cardiometabolic risk.

\section{Tolerability of HIIT in patients}

The trials published to date illustrate the tolerability of HIIT among diverse clinical populations and with varying study durations (see Table 1). Although large-scale trials are lacking, attempts have been made to assess the palatability of HIIT in previously sedentary populations. A group of obese women, some with type 2 diabetes, were noted to prefer a HIIT approach to MICT [90], as did volunteers with coronary heart disease [85]. Within HIIT protocols, enjoyment decreases with increasing interval length [91]. Specifically, intervals of 30 or $60 \mathrm{~s}$ resulted in greater enjoyment than $120 \mathrm{~s}$ intervals.

Good adherence to free-living, non-supervised HIIT ( $<3$ months) has been demonstrated in those with type 2 diabetes [38], NAFLD [39] and those with either impaired glucose tolerance or impaired fasting glucose [92]. Good adherence was also observed with interval walking ( 3 min alternative fast and slow walking) in a free-living environment over 4 months in patients with type 2 diabetes [36] and over 22 months in older adults [93]. Additional longer-term studies are required but, nonetheless, these results indicate good adherence and tolerability to independent HIIT exercise.

\section{Considerations when prescribing HIIT}

Beyond the plethora of specific protocols to choose from, the way in which HIIT protocols are often described is, in itself, a barrier to clinical implementation. The text box, 'Recommendations for HIIT prescription' provides a summary of our recommendations for prescribing HIIT in a clinical setting.
In research, HIIT is most commonly carried out as three sessions per week (Table 1). Such a frequency is consistent with the probable duration of the metabolic effects observed. As previously mentioned, the duration of intervals also varies from 1-4 min (see Table 1). Since longer intervals have not been conclusively shown to yield better clinical outcomes but have been shown to reduce enjoyment [91], it makes sense to start at the shorter end of the range. The accumulated time at highintensity during HIIT has varied from 10-20 min; starting at the lower end of this range allows for greater progression. Likewise, ratios of interval:recovery time also vary, with a 1:1 ratio offering a simple starting point. Last, keeping the intensity of the recovery period to a minimum is likely to increase enjoyment, at least initially.

Intensity is commonly measured using $\mathrm{HR}_{\max }$. At first glance, heart rate appears like a feasible option given the relative ubiquity of heart rate monitors. However, heart rate rises across intervals, as shown in Fig. 1. In our research, we have adopted a very practical approach, using the Rate of Perceived Exertion (RPE) 6-20 Borg scale as a guide of intensity; as previously reported, participants were asked to work at a 16-17 on the scale (or 'very hard') during each interval (Fig. 1) [38, 39]. RPE is an accurate predictor of exercise intensity in diabetes patients [94], however, like heart rate, it does have its limitations. Agreement between RPE and more objective measures of intensity is known to suffer both interand intra-individual variation. For example, when RPE was assessed during a set workload protocol, RPE increased from the first to the last interval [12]. Thus, using RPE trades some objectivity, but the benefit is a great deal of practicality.

Exercise selection is ultimately limited by what is available to the patient. Since peripheral metabolic adaptions are limited to the muscles undergoing forceful contractions during exercise [29], it is preferable to choose activities involving a large muscle mass. 


\section{What's next for HIIT?}

HIIT leads to modest improvements in metabolic control and weight loss. This is in contrast to calorie restriction, which leads to significant weight loss and improvements in metabolic control [40]. Combining HIIT with calorie restriction would accrue the cardiac benefits of HIIT and the weight loss benefits of calorie restriction. Additionally, exercise and calorie restriction together have been shown to improve glucose regulation by two-fold compared with the same amount of weight loss induced by exercise or calorie restriction alone [95]. Thus, there may be additive benefits for metabolic control if HIIT was used adjunct with energy restriction.

The myriad of different HIIT protocols adopted in the literature needs to be addressed. A standardised and consistent approach for prescribing HIIT protocols is missing, making it difficult to detect dose-response effects and the thresholds necessary to elicit desired changes. Most clinical HIIT studies have been short term ( $<4$ months, see Table 1$)$ and performed in a laboratory setting. The feasibility, acceptability and efficacy of longer-term HIIT in a real world setting requires investigation before it can be accepted as an alternative therapy for those with elevated cardiometabolic risk.

\section{Conclusion}

In circumstances where HIIT is not feasible, considered potentially unsafe or not well tolerated by an individual, MICT is effective at eliciting important health benefits. However, throughout this review we have shown that, provided unstable cardiovascular disease is excluded, HIIT appears to have a good safety profile and is well tolerated. Compared with other forms of exercise training, the use of HIIT for improving metabolic control and inducing weight loss should not be overstated. However, there are strong positive cardiovascular adaptations to HIIT that confer benefit to a population at risk of cardiac complications and therein lies the importance of HIIT for metabolic disease management. For optimal clinical benefit (improved glycaemic control and cardiovascular function), the value of HIIT appears likely to be adjunct to energy restriction, allowing HIIT to certainly make a hit.

Acknowledgements We thank L. Taylor (MoveLab, Newcastle University, Newcastle, UK) for assistance with creating the images for this manuscript.

Funding MIT was supported by a Senior Fellowship from the National Institute for Health Research.

Duality of interest The authors declare that there is no duality of interest associated with this manuscript.
Contribution statement All authors were involved in drafting the article and revising it critically for important intellectual content, and approving the final version for publication.

Open Access This article is distributed under the terms of the Creative Commons Attribution 4.0 International License (http:// creativecommons.org/licenses/by/4.0/), which permits unrestricted use, distribution, and reproduction in any medium, provided you give appropriate credit to the original author(s) and the source, provide a link to the Creative Commons license, and indicate if changes were made.

\section{References}

1. Cordain L, Gotshall R, Eaton S, Eaton S (1998) Physical Activity, Energy Expenditure and Fitness: An Evolutionary Perspective. Int J Sports Med 19:328-335

2. WHO (2014) The top 10 causes of death. Fact sheet no. 310. WHO, Geneva

3. Inzucchi SE, Bergenstal RM, Buse JB et al (2015) Management of hyperglycaemia in type 2 diabetes, 2015: a patient-centered approach. Update to a position statement of the American Diabetes Association and the European association for the Study of Diabetes. Diabetologia 58:429-442

4. Jensen MD, Ryan DH, Apovian CM et al (2014) 2013 AHA/ACC/ TOS guideline for the management of overweight and obesity in adults: a report of the American College of Cardiology/American Heart Association Task Force on Practice Guidelines and The Obesity Society. Circulation 129:S102-S138

5. Colberg SR, Sigal RJ, Fernhall B et al (2010) Exercise and type 2 diabetes: the American College of Sports Medicine and the American Diabetes Association: joint position statement executive summary. Diabetes Care 33:2692-2696

6. ACSM (2014) ACSM's guidelines for exercise testing and prescription, 9th edn. Wolters Kluwer/Lippincott Williams \& Wilkins, Philadelphia

7. Jelleyman C, Yates T, O'Donovan G et al (2015) The effects of high-intensity interval training on glucose regulation and insulin resistance: a meta-analysis. Obes Rev 16:942-961

8. Weston KS, Wisløff U, Coombes JS (2014) High-intensity interval training in patients with lifestyle-induced cardiometabolic disease: a systematic review and meta-analysis. Br J Sports Med 48:1227-1234

9. Gibala MJ, Little JP, Macdonald MJ, Hawley JA (2012) Physiological adaptations to low-volume, high-intensity interval training in health and disease. J Physiol 590:1077-1084

10. Burgomaster KA, Hughes SC, Heigenhauser GJF et al (2005) Six sessions of sprint interval training increases muscle oxidative potential and cycle endurance capacity in humans. J Appl Physiol 98: 1985-1990

11. Fex A, Leduc-Gaudet J-P, Filion M-E et al (2015) Effect of elliptical high intensity interval training on metabolic risk factor in pre- and type 2 diabetes patients: a pilot study. J Phys Act Health 12:942-946

12. Little JP, Gillen JB, Percival ME et al (2011) Low-volume highintensity interval training reduces hyperglycemia and increases muscle mitochondrial capacity in patients with type 2 diabetes. J Appl Physiol 111:1554-1560

13. Ren JM, Semenkovich CF, Gulve EA et al (1994) Exercise induces rapid increases in GLUT4 expression, glucose transport capacity, and insulin-stimulated glycogen storage in muscle. J Biol Chem 269:14396-14401

14. Karstoft K, Winding K, Knudsen SH et al (2014) Mechanisms behind the superior effects of interval vs continuous training on 
glycaemic control in individuals with type 2 diabetes: a randomised controlled trial. Diabetologia 57:2081-2093

15. Ritov VB, Menshikova EV, Azuma K et al (2010) Deficiency of electron transport chain in human skeletal muscle mitochondria in type 2 diabetes mellitus and obesity. Am J Physiol Endocrinol Metab 298:E49-58

16. Schrauwen-Hinderling VB, Kooi ME, Hesselink MKC et al (2007) Impaired in vivo mitochondrial function but similar intramyocellular lipid content in patients with type 2 diabetes mellitus and BMImatched control subjects. Diabetologia 50:113-120

17. Mootha VK, Lindgren CM, Eriksson K-F et al (2003) PGC-1 $\alpha$ responsive genes involved in oxidative phosphorylation are coordinately downregulated in human diabetes. Nat Genet 34:267-273

18. Wu Z, Puigserver P, Andersson U et al (1999) Mechanisms controlling mitochondrial biogenesis and respiration through the thermogenic coactivator PGC-1. Cell 98:115-124

19. Little JP, Safdar A, Wilkin GP et al (2010) A practical model of lowvolume high-intensity interval training induces mitochondrial biogenesis in human skeletal muscle: potential mechanisms. J Physiol 588:1011-1022

20. Tjønna AE, Lee SJ, Rognmo Ø et al (2008) Aerobic interval training versus continuous moderate exercise as a treatment for the metabolic syndrome: A pilot study. Circulation 118:346-354

21. Daussin FN, Zoll J, Dufour SP et al (2008) Effect of interval versus continuous training on cardiorespiratory and mitochondrial functions: relationship to aerobic performance improvements in sedentary subjects. Am J Physiol Regul Integr Comp Physiol 295:R264-R272

22. Little JP, Jung ME, Wright AE et al (2014) Effects of high-intensity interval exercise versus continuous moderate-intensity exercise on postprandial glycemic control assessed by continuous glucose monitoring in obese adults. Appl Physiol Nutr Metab 39:835-841

23. Gillen JB, Little JP, Punthakee Z et al (2012) Acute high-intensity interval exercise reduces the postprandial glucose response and prevalence of hyperglycaemia in patients with type 2 diabetes. Diabetes Obes Metab 14:575-577

24. Terada T, Wilson BJ, Myette-Cóté E et al (2016) Targeting specific interstitial glycemic parameters with high-intensity interval exercise and fasted-state exercise in type 2 diabetes. Metabolism 65:599-608

25. Karstoft K, Christensen CS, Pedersen BK, Solomon TPJ (2014) The acute effects of interval- vs continuous-walking exercise on glycemic control in subjects with type 2 diabetes: A crossover, controlled study. J Clin Endocrinol Metab 99:3334-3342

26. Tjønna AE, Rognmo Ø, Bye A et al (2011) Time course of endothelial adaptation after acute and chronic exercise in patients with metabolic syndrome. J Strength Cond Res 25:2552-2558

27. Cavalot F, Pagliarino A, Valle M et al (2011) Postprandial blood glucose predicts cardiovascular events and all-cause mortality in type 2 diabetes in a 14-year follow-up: lessons from the San Luigi Gonzaga Diabetes Study. Diabetes Care 34:2237-2243

28. Ceriello A (2005) Postprandial hyperglycemia and diabetes complications: is it time to treat? Diabetes 54:1-7

29. Frøsig C, Richter EA (2009) Improved insulin sensitivity after exercise: focus on insulin signaling. Obesity (Silver Spring) 17(Suppl 3):S15-S20

30. Mikines KJ, Sonne B, Farrell PA et al (1988) Effect of physical exercise on sensitivity and responsiveness to insulin in humans. Am J Physiol Endocrinol Metab 254:248-259

31. Gollnick PD, Piehl K, Saltin B (1974) Selective glycogen depletion pattern in human muscle fibres after exercise of varying intensity and at varying pedalling rates. J Physiol 241:45-57

32. America Diabetes Association (2014) Standards of Medical Care in Diabetes-2014. Diabetes Care 37(Suppl 1):S14-S80

33. Earnest CP, Lupo M, Thibodaux J et al (2013) Interval training in men at risk for insulin resistance. Int J Sports Med 34:355-363
34. Madsen SM, Thorup AC, Overgaard K, Jeppesen PB (2015) High intensity interval training improves glycaemic control and pancreatic $\beta$ cell function of type 2 diabetes patients. PLoS One 10:1-24

35. Stensvold D, Tjønna AE, Skaug E-A et al (2010) Strength training versus aerobic interval training to modify risk factors of metabolic syndrome. J Appl Physiol 108:804-810

36. Karstoft K, Winding K, Knudsen $\mathrm{S}$ et al (2013) The effects of freeliving interval-walking training on glycemic control, body composition, and physical fitness in type 2 diabetic patients. Diabetes Care 36:228-236

37. Shaban N, Kenno K, Milne K (2014) The effects of a 2 week modified high intensity interval training program on the homeostatic model of insulin resistance (HOMA-IR) in adults with type 2 diabetes. J Sports Med Phys Fitness 54:203-209

38. Cassidy S, Thoma C, Hallsworth K et al (2016) High intensity intermittent exercise improves cardiac structure and function and reduces liver fat in patients with type 2 diabetes: a randomised controlled trial. Diabetologia 59:56-66

39. Hallsworth K, Thoma C, Hollingsworth KG et al (2015) Modified high-intensity interval training reduces liver fat and improves cardiac function in non-alcoholic fatty liver disease: a randomized controlled trial. Clin Sci (Lond) 129:1097-1105

40. Lim EL, Hollingsworth KG, Aribisala BS et al (2011) Reversal of type 2 diabetes: normalisation of beta cell function in association with decreased pancreas and liver triacylglycerol. Diabetologia 54: 2506-2514

41. Mitranun W, Deerochanawong C, Tanaka H, Suksom D (2014) Continuous vs interval training on glycemic control and macro- and microvascular reactivity in type 2 diabetic patients. Scand J Med Sci Sports 24:e69-e76

42. ACSM (2005) ACSM's resource manual for guidelines for exercise testing and prescription, 5th edn. Lippincott Williams and Wilkins, Philadelphia

43. Simoneau JA, Colberg SR, Thaete FL, Kelley DE (1995) Skeletal muscle glycolytic and oxidative enzyme capacities are determinants of insulin sensitivity and muscle composition in obese women. FASEB J 9:273-278

44. Hollekim-Strand SM, Bjørgaas MR, Albrektsen G et al (2014) Highintensity interval exercise effectively improves cardiac function in patients with type 2 diabetes mellitus and diastolic dysfunction: a randomized controlled trial. J Am Coll Cardiol 64:1758-1760

45. Robinson E, Durrer C, Simtchouk S et al (2015) Short-term highintensity interval and moderate-intensity continuous training reduce leukocyte TLR4 in inactive adults at elevated risk of type 2 diabetes. J Appl Physiol 110:508-516

46. Terada T, Friesen A, Chahal BS et al (2013) Feasibility and preliminary efficacy of high intensity interval training in type 2 diabetes. Diabetes Res Clin Pract 99:120-129

47. Thomas D, Elliott E, Naughton G (2006) Exercise for type 2 diabetes mellitus (Review). Cochrane Database Syst Rev 19:CD002968

48. Johansen K (1999) Efficacy of metformin in the treatment of NIDDM. Meta-analysis. Diabetes Care 22:33-37

49. Rafiq N, Bai C, Fang Y et al (2009) Long-term follow-up of patients with nonalcoholic fatty liver. Clin Gastroenterol Hepatol 7:234-238

50. Garcia MJ, McNamara PM, Gordon T, Kannel WB (1974) Morbidity and mortality in diabetics in the Framingham population. Sixteen year follow-up study. Diabetes 23:105-111

51. Hasenfuss G (1998) Animal models of human cardiovascular disease, heart failure and hypertrophy. Cardiovase Res 39:60-76

52. Wisløff U, Ellingsen $\varnothing$, Kemi OJ (2009) High-intensity interval training to maximize cardiac benefits of exercise training? Exerc Sport Sci Rev 37:139-146

53. Stølen TO, Høydal MA, Kemi OJ et al (2009) Interval training normalizes cardiomyocyte function, diastolic $\mathrm{Ca}^{2+}$ control, and $\mathrm{SR} \mathrm{Ca}^{2+}$ release synchronicity in a mouse model of diabetic cardiomyopathy. Circ Res 105:527-536 
54. Wisløff U, Loennechen JP, Currie S et al (2002) Aerobic exercise reduces cardiomyocyte hypertrophy and increases contractility, $\mathrm{Ca}^{2+}$ sensitivity and SERCA-2 in rat after myocardial infarction. Cardiovasc Res 54:162-174

55. Kemi OJ, Haram PM, Loennechen JP et al (2005) Moderate vs. high exercise intensity: differential effects on aerobic fitness, cardiomyocyte contractility, and endothelial function. Cardiovasc Res 67:161-172

56. Zile MR, Gottdiener JS, Hetzel SJ et al (2011) Prevalence and significance of alterations in cardiac structure and function in patients with heart failure and a preserved ejection fraction. Circulation 124:2491-2501

57. Rijzewijk LJ, van der Meer RW, Lamb HJ et al (2009) Altered myocardial substrate metabolism and decreased diastolic function in nonischemic human diabetic cardiomyopathy: studies with cardiac positron emission tomography and magnetic resonance imaging. J Am Coll Cardiol 54:1524-1532

58. Frey N, Katus HA, Olson EN, Hill JA (2004) Hypertrophy of the heart: a new therapeutic target? Circulation 109:1580-1589

59. Molmen-Hansen HE, Stolen T, Tjonna AE et al (2012) Aerobic interval training reduces blood pressure and improves myocardial function in hypertensive patients. Eur J Prev Cardiol 19:151-160

60. Wisløff U, Støylen A, Loennechen JP et al (2007) Superior cardiovascular effect of aerobic interval training versus moderate continuous training in heart failure patients: a randomized study. Circulation 115:3086-3094

61. Coletta AP, Cleland JGF, Freemantle N, Clark AL (2004) Clinical trials update from the European Society of Cardiology Heart Failure meeting: SHAPE, BRING-UP 2 VAS, COLA II, FOSIDIAL, BETACAR, CASINO and meta-analysis of cardiac resynchronisation therapy. Eur J Heart Fail 6:673-676

62. Lumens J, Delhaas T, Arts T et al (2006) Impaired subendocardial contractile myofiber function in asymptomatic aged humans, as detected using MRI. Am J Physiol Heart Circ Physiol 291: H1573-H1579

63. Goland S, Shimoni S, Zornitzki Tet al (2006) Cardiac abnormalities as a new manifestation of nonalcoholic fatty liver disease: echocardiographic and tissue Doppler imaging assessment. J Clin Gastroenterol 40:949-955

64. Halley CM, Houghtaling PL, Khalil MK et al (2011) Mortality rate in patients with diastolic dysfunction and normal systolic function. Arch Intern Med 171:1082-1087

65. Avogaro A, Albiero M, Menegazzo L et al (2011) Endothelial dysfunction in diabetes: the role of reparatory mechanisms. Diabetes Care 34(Suppl 2):S285-S290

66. Ramos JS, Dalleck LC, Tjonna AE et al (2015) The impact of highintensity interval training versus moderate-intensity continuous training on vascular function: a systematic review and meta-analysis. Sports Med 45:679-692

67. Pescatello LS, Franklin BA, Fagard R et al (2004) Exercise and hypertension. Med Sci Sports Exerc 36:533-553

68. Barry VW, Baruth M, Beets MW et al (2014) Fitness vs. fatness on all-cause mortality: a meta-analysis. Prog Cardiovasc Dis 56:382-390

69. Sui X, Hooker SP, Lee I-M et al (2008) A prospective study of cardiorespiratory fitness and risk of type 2 diabetes in women. Diabetes Care 31:550-555

70. Blair SN, Kohl HW, Barlow CE et al (1995) Changes in physical fitness and all-cause mortality. A prospective study of healthy and unhealthy men. JAMA 273:1093-1098

71. Erikssen G, Liestøl K, Bjørnholt J et al (1998) Changes in physical fitness and changes in mortality. Lancet 352:759-762

72. Milanović Z, Sporiš G, Weston M (2015) Effectiveness of highintensity interval training (HIT) and continuous endurance training for $\mathrm{VO}_{2 \max }$ improvements: a systematic review and meta-analysis of controlled trials. Sports Med 45:1469-1481
73. Liou K, Ho S, Fildes J, Ooi S-Y (2016) High intensity interval versus moderate intensity continuous training in patients with coronary artery disease: a meta-analysis of physiological and clinical parameters. Heart Lung Circ 25:166-174

74. Kaminsky LA, Arena R, Beckie TM et al (2013) The importance of cardiorespiratory fitness in the United States: the need for a national registry: a policy statement from the American Heart Association. Circulation 127:652-662

75. Bækkerud FH, Solberg F, Leinan IM et al (2016) Comparison of three popular exercise modalities on $\mathrm{VO}_{2 \max }$ in overweight and obese. Med Sci Sports Exerc 48:491-498

76. Sjöström CD, Lissner L, Wedel H, Sjöström L (1999) Reduction in incidence of diabetes, hypertension and lipid disturbances after intentional weight loss induced by bariatric surgery: the SOS Intervention Study. Obes Res 7:477-484

77. Heydari M, Freund J, Boutcher SH (2012) The effect of highintensity intermittent exercise on body composition of overweight young males. J Obes 2012:480467

78. Fisher G, Brown AW, Bohan Brown MM et al (2015) High intensity interval- vs moderate intensity-training for improving cardiometabolic health in overweight or obese males: a randomized controlled trial. PLoS One 10:1-15

79. Nakamura T, Tokunaga K, Shimomura I et al (1994) Contribution of visceral fat accumulation to the development of coronary artery disease in non-obese men. Atherosclerosis 107:239-246

80. Fujioka S, Matsuzawa Y, Tokunaga K, Tarui S (1987) Contribution of intra-abdominal fat accumulation to the impairment of glucose and lipid metabolism in human obesity. Metabolism 36:54-59

81. Talanian JL, Galloway SDR, Heigenhauser GJF et al (2007) Two weeks of high-intensity aerobic interval training increases the capacity for fat oxidation during exercise in women. J Appl Physiol 102:1439-1447

82. Zouhal H, Jacob C, Delamarche P, Gratas-Delamarche A (2008) Catecholamines and the effects of exercise, training and gender. Sports Med 38:401-423

83. Rebuffé-Scrive M, Andersson B, Olbe L, Björntorp P (1989) Metabolism of adipose tissue in intraabdominal depots of nonobese men and women. Metabolism 38:453-458

84. Sim AY, Wallman KE, Fairchild TJ, Guelfi KJ (2014) Highintensity intermittent exercise attenuates ad-libitum energy intake. Int J Obes 38:417-422

85. Guiraud T, Nigam A, Juneau M et al (2011) Acute responses to high-intensity intermittent exercise in CHD patients. Med Sci Sports Exerc 43:211-217

86. Guiraud T, Juneau M, Nigam A et al (2010) Optimization of high intensity interval exercise in coronary heart disease. Eur J Appl Physiol 108:733-740

87. Meyer K, Samek L, Schwaibold M et al (1996) Physical responses to different modes of interval exercise in patients with chronic heart failure - application to exercise training. Eur Heart J 17:1040-1047

88. Rognmo Ø, Moholdt T, Bakken $\mathrm{H}$ et al (2012) Cardiovascular risk of high- versus moderate-intensity aerobic exercise in coronary heart disease patients. Circulation 126:1436-1440

89. Thompson PD, Franklin BA, Balady GJ et al (2007) Exercise and acute cardiovascular events placing the risks into perspective: a scientific statement from the American Heart Association Council on Nutrition, Physical Activity, and Metabolism and the Council on Clinical Cardiology. Circulation 115:2358-2368

90. Coquart JBJ, Lemaire C, Dubart A-E et al (2008) Intermittent versus continuous exercise: effects of perceptually lower exercise in obese women. Med Sci Sports Exerc 40:1546-1553

91. Martinez N, Kilpatrick MW, Salomon K et al (2015) Affective and enjoyment responses to high-intensity interval training in overweight-to-obese and insufficiently active adults. J Sport Exerc Psychol 37:138-149 
92. Jung ME, Bourne JE, Beauchamp MR et al (2015) High-intensity interval training as an efficacious alternative to moderate-intensity continuous training for adults with prediabetes. J Diabetes Res 2015:1-9

93. Masuki S, Mori M, Tabara Y et al (2015) The factors affecting adherence to a long-term interval walking training program in middle-aged and older people. J Appl Physiol 118:595-603
94. Colberg SR, Swain DP, Vinik AI (2003) Use of heart rate reserve and rating of perceived exertion to prescribe exercise intensity in diabetic autonomic. Diabetes Care 26:986-990

95. Weiss EP, Albert SG, Reeds DN et al (2015) Calorie restriction and matched weight loss from exercise: independent and additive effects on glucoregulation and the incretin system in overweight women and men. Diabetes Care 38:1253-1262 